\title{
Modeling and flat control law for a fine pointing system based on semi active magnetic bearings
}

\author{
Vincent Mahout, Xavier Prats \\ LAAS/CNRS \\ 7, avenue du colonel Roche, 31077 Toulouse \\ mahout@laas.fr, prats@space.com \\ Jean Mignot \\ CNES \\ 18, avenue Edouard Belin, BP 142131401 Toulouse \\ jean.mignot@cnes.fr
}

\begin{abstract}
-
In this paper aspects of non linear systems and fiat control are studied for a specific application of a satellite fine pointing breadboard based on semi active magnetic bearings actuators. Authors propose a complete 6 degrees of freedom mechanical model which describes the system dynamics. A completely non linear and unstable system is obtained leading to implement non linear control laws. A combination of flat control, which ensures trajectory tracking and path planning, with a linear controller which rejects uncertainties and additive noise is implemented and simulated. Simulations proves the efficiency of control law, leading to a system which is stable and reaching the high precision performances desired for the application.
\end{abstract}

Keywords: Nonlinear Systems, Flat Control, Modeling, Satellite, Magnetic Bearing.

\section{INTRODUCTION}

In the last decades, studies in non linear control theory have been developed in order to deal with non linear systems, obtaining excellent results and better performances than linear classical control approaches. In this way, new non linear techniques have appeared in fields like filtering, observer and prediction design, control laws synthesizing, etc.

This paper deals with the study of a non linear control law applied in a satellite pointing breadboard. This system performs atmosphere analysis by a scanning rotation of a mirror attached to a shaft. A DC motor is charged to give this rotating motion to the shaft. Two sets of semi active magnetic bearings assure rotor axial and radial stability, supporting it by magnetic levitation.

Previous studies have been done, splitting up the model of the system into a rotating motion and into a shaft levitation. This approach did not consider mechanical couplings among these movements and system performances were not as expected.

Authors propose to redo system model taking into account all mechanical couplings neglected in the old models. Flat control is proposed to control the non linear resulting system. Studies on flat control in active magnetic bearings could be found in [4]. In this paper, semi active magnetic bearings are studied which means a more complex and unstable model compared with only active magnetic bearings systems.

The first section of this paper proposes a new 6 degrees of freedom (DOF) system model. The second section develops a non linear control law, based on flat control, designed to give to the shaft the desired scan rotation angle. Finally, the third section presents simulation results.

\section{MODELING}

In order to find state space equations, which will describe shaft and mirror dynamics, a first study of magnetic bearings actuators must be obtained to model the forces and the moments applied on the shaft. The maximimum rotation is about 120 degrees.

\section{A. The semi active magnetic bearing}

Scan mechanism is composed by two sets of magnetic bearings, MBA and MBB, which ensures shaft levitation. MBA (for magnetic bearing A) is a 3 DOF magnetic bearing, ensuring radial and axial levitation while MBB (magnetic bearing $B$ ) is a 2 DOF magnetic bearing, so ensuring only radial levitation. These bearings are in fact semi active magnetic bearings, which means that internal magnetic fields are generated by passive elements (magnets) plus active or control elements (current coils).

In a 1 DOF simplification a semi active magnetic bearing produces two kinds of forces towards rotor shaft. First force, which is unstable, is generated by the magnet, and can be linearized as:

$$
f^{S} \simeq-k^{S} \varepsilon
$$

where $\varepsilon$ is the air gap between the rotor and the stator of the actuator and $k^{S}$ is just a constant. This force is called "negative stiffness force" since it is equivalent to the force generated by a spring with a imaginary "negative" stiffness, that is a unstable force.

Second force is generated by current coils and in the same way, it can be linearized as: 


$$
f^{C} \simeq k^{C} i
$$

where $i$ is coil current and $k^{C}$ is a constant. This force is called "control force" and it will be used to stabilize shaft movements using a position measure feedback.

Generalization for a 2 and 3 DOF magnetic bearing is immediate and leads to a total of 5 control currents which will become system actuators for properly shaft stabilization.

\section{B. Hypothesis}

Some hypothesis should be considered for further modeling study. Principal ones are:

$\mathcal{H} 1$ : Rotor shaft is assumed to be a rigid body.

$\mathcal{H} 2$ : Parasite rotation angles are small.

$\mathcal{H} 3$ : Output noise quantifications and measurement noise are not taken into account.

\section{Body mechanics}

In order to locate at each time instant the system formed by the mirror and the shaft, a vector $\mathbf{r}(t)$ is used to describe its translation, and another vector $\theta(t)$ describes its rotation. Two reference frames are defined. $S F$ is the stator frame, which corresponds to a world space fixed frame. $R F$ is the rotor frame, which follows body movements. Figure 1 illustrates these two cartesian reference systems.

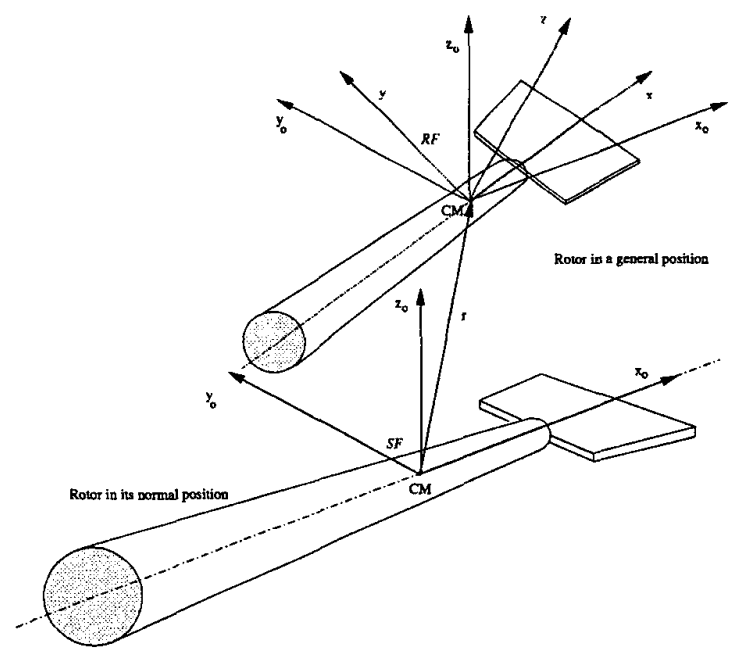

Fig. 1. Definition of Stator Frame (SF) and Rotor Frame (RF)

Then, $\mathbf{r}(\mathbf{t})$ is composed by the three simple translations of body center of mass:

$$
\mathbf{r}(\mathbf{t}) \triangleq\left[\begin{array}{lll}
x(t) & y(t) & z(t)
\end{array}\right]^{\mathbf{T}}
$$

Rotation $\theta(\mathbf{t})$ is defined as a common Euler angle $i-j-k$ sequence:

$$
\theta(t) \triangleq\left[\begin{array}{lll}
\alpha(t) & \beta(t) & \delta(t)
\end{array}\right]^{\mathbf{T}}
$$

being $\alpha$ (scan angle), $\beta$ and $\delta$ (parasite angles) these three Euler angles.

Hence, rotation matrix which transforms a fixed point in the body space into the world space is given by [6]:

$$
\mathcal{R} \simeq\left[\begin{array}{ccc}
1 & -\delta \cos \alpha+\beta \sin \alpha & \delta \sin \alpha+\beta \cos \alpha \\
\delta & \cos \alpha & -\sin \alpha \\
-\beta & \sin \alpha & \cos \alpha
\end{array}\right]
$$

where trigonometric expressions involving $\beta$ and $\delta$ are being simplified (according to hypothesis $\mathcal{H} \mathbf{2}$ ).

\section{C.1 Linear and angular velocity}

Since $\mathbf{r}(\mathbf{t})$ is the position of the center of mass in world space, $\dot{\mathbf{r}}$ is the velocity of the center of mass in world space. Then, linear velocity $\mathbf{v}(\mathbf{t})$ is defined as:

$$
\mathbf{v}(\mathbf{t}) \triangleq\left[\begin{array}{lll}
v_{x}(t) & v_{y}(t) & v_{z}(t)
\end{array}\right]^{\mathbf{T}}=\dot{\mathbf{r}}
$$

In addition to translating, body can also spin. Then, angular velocity $\omega(\mathbf{t})$ is defined as

$$
\omega(\mathbf{t}) \triangleq\left[\begin{array}{lll}
p(t) & q(t) & r(t)
\end{array}\right]^{\mathbf{T}}
$$

and it is related with the temporal variation of Euler angles by:

$$
\dot{\theta}=\mathcal{T} \cdot \omega
$$

According again with hypothesis $\mathcal{H} 2, \mathcal{T}$ matrix can be approximated as:

$$
\mathcal{T} \simeq\left[\begin{array}{ccc}
1 & \delta & 0 \\
-\delta & 1 & 0 \\
\beta & 0 & 1
\end{array}\right]
$$

\section{C.2 Body dynamics}

Applying fundamental dynamics law in body translation we have:

$$
m \dot{\mathbf{v}}(\mathbf{t})=\sum_{\mathbf{i}} \mathbf{F}_{\mathbf{i}}(\mathbf{t})
$$

being $m$ body mass and $\sum_{i} \mathbf{F}_{\mathbf{i}}(\mathbf{t})$ the total external force acting on the body at time $t$.

Defining $\mathbf{L}(\mathbf{t})$ as body angular momentum and applying again fundamental dynamics laws in rotation:

$$
\dot{\mathrm{L}}(\mathbf{t})=\sum_{\mathbf{i}} \mathbf{M}_{\mathbf{i}}(\mathbf{t})
$$

being $\sum_{i} \mathbf{M}_{\mathbf{i}}(\mathbf{t})$ the total external torque acting on the body at time $t$.

Introducing $\mathcal{I}_{\mathbf{b}}$ as the body inertia tensor specified in body-space (thus, constant over time) we have:

$$
\mathbf{L}(\mathbf{t})=\mathcal{R}(\mathbf{t}) \cdot \mathcal{I}_{\mathbf{b}} \cdot \mathcal{R}^{\mathbf{T}}(\mathbf{t}) \cdot \omega(\mathbf{t})
$$

Writing again equation (11) we obtain finally: 


$$
\begin{aligned}
& \sum_{i} \mathbf{M}_{\mathbf{i}}(\mathbf{t})=\dot{\mathcal{R}}(\mathbf{t}) \cdot \mathcal{I}_{\mathbf{b}} \cdot \mathcal{R}^{\mathbf{T}}(\mathbf{t}) \cdot \omega(\mathbf{t})+ \\
& +\mathcal{R}(\mathbf{t}) \cdot \mathcal{I}_{\mathbf{b}} \cdot \dot{\mathcal{R}}^{\mathbf{T}}(\mathbf{t}) \cdot \omega(\mathbf{t})+\mathcal{R}(\mathbf{t}) \cdot \mathcal{I}_{\mathbf{b}} \cdot \mathcal{R}^{\mathbf{T}}(\mathbf{t}) \cdot \dot{\omega}(\mathbf{t})
\end{aligned}
$$

\section{State space equations}

We need to develop $\sum_{i} \mathbf{F}_{\mathbf{i}}$ and $\sum_{i} \mathbf{M}_{\mathbf{i}}$ terms in order to obtain a complete set of state equations (time dependence is omitted in following notation for further clarity).

The total external force acting on the shaft is the sum of the set of negative stiffness forces and the set of control forces (see section II-A). According to equations (1) and (2) the total external force is:

$$
\begin{aligned}
\sum \mathbf{F}_{\mathbf{i}}= & {\left[\begin{array}{c}
f_{x_{A}} \\
f_{y_{A}}+f_{y_{B}} \\
f_{z_{A}}+f_{z_{B}}
\end{array}\right]=\left[\begin{array}{c}
k_{x_{A}}^{R} \varepsilon_{x_{A}} \\
k_{y_{A}}^{R} \varepsilon_{y_{A}}+k_{y_{B}}^{R} \varepsilon_{y_{B}} \\
k_{z_{A}}^{R} \varepsilon_{z_{A}}+k_{z_{B}}^{R} \varepsilon_{z_{B}}
\end{array}\right]+} \\
& +\left[\begin{array}{cccccc}
k_{x_{A}}^{C} & 0 & 0 & 0 & 0 & 0 \\
0 & k_{y_{A}}^{C} & 0 & k_{y_{B}}^{C} & 0 & 0 \\
0 & 0 & k_{z_{A}}^{C} & 0 & k_{z_{B}}^{C} & 0
\end{array}\right] \cdot \mathbf{u}
\end{aligned}
$$

where control input $\mathbf{u}$ is formed by 5 magnetic bearing control currents plus the current $i_{m}$ of the DC motor charged to give the rotating motion to the shaft.

$$
\mathbf{u} \triangleq\left[\begin{array}{llllll}
i_{x_{A}} & i_{y_{A}} & i_{z_{A}} & i_{y_{B}} & i_{z_{B}} & i_{m}
\end{array}\right]^{\mathbf{T}}
$$

Total external torque is the torque created by those forces plus the torque created by the motor. Therefore:

$$
\sum \mathbf{M}_{\mathbf{i}}=\left[\begin{array}{c}
0 \\
f_{z_{A}} l_{A}-f_{z_{B}} l_{B} \\
-f_{y_{A}} l_{A}+f_{y_{B}} l_{B}
\end{array}\right]+\mathcal{R} \cdot\left[\begin{array}{c}
\tau_{m} \\
0 \\
0
\end{array}\right]
$$

where $l_{A}$ (and $l_{B}$ ) is the distance of MBA (and MBB) from body center of mass and $\tau_{m}$ is motor torque.

Air gaps, in a first order approximation, are given by:

$$
\begin{gathered}
\varepsilon_{x_{A}}=x \\
\varepsilon_{y_{A}}=y-\delta l_{A} \quad \varepsilon_{y_{B}}=y+\delta l_{B} \\
\varepsilon_{z_{A}}=z+\beta l_{A} \quad \varepsilon_{z_{B}}=z-\beta l_{B}
\end{gathered}
$$

In order to simplify the notations, we can define:

$$
\begin{gathered}
\mathcal{M}(\theta) \triangleq \mathcal{R} \cdot \mathcal{I}_{\mathbf{b}}^{-1} \cdot \mathcal{R}^{\mathbf{T}} \\
\mathcal{N}(\theta, \omega) \triangleq \dot{\mathcal{R}} \cdot \mathcal{I}_{\mathbf{b}} \cdot \mathcal{R}^{\mathbf{T}}+\mathcal{R} \cdot \mathcal{I}_{\mathbf{b}} \cdot \dot{\mathcal{R}}^{\mathbf{T}} \\
\mathbf{V}_{\mathbf{1}}(\mathbf{r}, \theta) \triangleq\left[\begin{array}{c}
k_{x_{A}}^{R} \varepsilon_{x_{A}} \\
k_{y_{A}}^{R} \varepsilon_{y_{A}}+k_{y_{B}}^{R} \varepsilon_{y_{B}} \\
k_{z_{A}}^{R} \varepsilon_{z_{A}}+k_{z_{B}}^{R} \varepsilon_{z_{B}}
\end{array}\right] \\
\mathbf{V}_{\mathbf{2}}(\mathbf{r}, \theta) \triangleq\left[\begin{array}{c}
0 \\
\left(k_{z_{A}}^{R} \varepsilon_{z_{A}}\right) l_{A}-\left(k_{z_{B}}^{R} \varepsilon_{z_{B}}\right) l_{B} \\
-\left(k_{y_{A}}^{R} \varepsilon_{y_{A}}\right) l_{A}+\left(k_{y_{B}}^{R} \varepsilon_{y_{B}}\right) l_{B}
\end{array}\right]
\end{gathered}
$$

$$
\begin{gathered}
\mathcal{K}_{\mathbf{f}}^{\mathbf{C}} \triangleq\left[\begin{array}{cccccc}
k_{x_{A}}^{C} & 0 & 0 & 0 & 0 & 0 \\
0 & k_{y_{A}}^{C} & 0 & k_{y_{B}}^{C} & 0 & 0 \\
0 & 0 & k_{z_{A}}^{C} & 0 & k_{z_{B}}^{C} & 0
\end{array}\right] \\
\mathcal{K}_{\mathbf{m}}^{\mathbf{C}} \triangleq\left[\begin{array}{cccccc}
0 & 0 & 0 & 0 & 0 & 0 \\
0 & 0 & k_{z_{A}}^{C} l_{A} & 0 & -k_{z_{B}}^{C} l_{B} & 0 \\
0 & -k_{y_{A}}^{C} l_{A} & 0 & k_{y_{B}}^{C} l_{B} & 0 & 0
\end{array}\right] \\
\mathcal{K}_{\mathbf{m}_{\mathbf{m}}}^{\mathbf{C}} \triangleq\left[\begin{array}{cccccc}
0 & 0 & 0 & 0 & 0 & K m \\
0 & 0 & 0 & 0 & 0 & 0 \\
0 & 0 & 0 & 0 & 0 & 0
\end{array}\right] \\
\mathcal{S}(\theta)=\mathcal{M}(\theta) \cdot\left[\mathcal{K}_{\mathbf{m}}^{\mathbf{C}}+\mathcal{R} \cdot \mathcal{K}_{\mathbf{m}_{\mathrm{m}}}^{\mathbf{C}}\right]
\end{gathered}
$$

The final state space equations are:

$$
\left\{\begin{aligned}
\dot{\mathbf{r}}= & \mathbf{v} \\
\dot{\theta}= & \mathcal{T}(\theta) \cdot \omega \\
\dot{\mathbf{v}}= & \frac{1}{\mathrm{~m}} \mathbf{V}_{\mathbf{1}}(\mathbf{r}, \theta)+\frac{1}{\mathrm{~m}} \mathcal{K}_{\mathbf{f}}^{\mathbf{C}} \cdot \mathbf{u} \\
\dot{\omega}= & \mathcal{M}(\theta) \cdot \mathbf{V}_{\mathbf{2}}(\mathbf{r}, \theta)-\mathcal{M}(\theta) \cdot \mathcal{N}(\theta, \omega) \cdot \omega+ \\
& +\mathcal{S}(\theta) \cdot \mathbf{u}
\end{aligned}\right.
$$

\section{Control LaWS}

The system model is completely non linear and can not be linearized because of the wide range of values for the angle $\alpha$ and the impossibility to avoid non linear mechanical couplings among the three rotation axis. Therefore a non linear control law has been developed, in particular, a flat control method.

\section{A. Introduction to flat control}

Flat control is a quite recent control theory. It has been developed by M. Fliess (see for example [1]). We can note that, in the case of SISO system, flat theory and exact linearization feedback [3], [5] become confused.

Consider a non linear model given by:

$$
\dot{\mathbf{x}}=f(\mathbf{x}, \mathbf{u})
$$

with state and control vectors defined as:

$$
\begin{aligned}
& \mathbf{x}=\left[\begin{array}{lll}
x_{1} & \cdots & x_{n}
\end{array}\right]^{T} \in \mathbb{R}^{n} \quad \text { and } \\
& \mathbf{u}=\left[\begin{array}{lll}
u_{1} & \cdots & u_{m}
\end{array}\right]^{T} \in \mathbb{R}^{m}
\end{aligned}
$$

System (27) is called a differential flat system if and only if there exists an system output $\mathbf{y} \in \mathbb{R}^{\mathbf{m}}$ such that:

$$
\left\{\begin{array}{c}
\mathbf{x}=\mathbf{A}\left(\mathbf{y}, \dot{\mathbf{y}}, \ddot{\mathbf{y}}, \cdots, \mathbf{y}^{(\alpha)}\right) \\
\mathbf{u}=\mathbf{B}\left(\mathbf{y}, \dot{\mathbf{y}}, \ddot{\mathbf{y}}, \cdots, \mathbf{y}^{(\alpha+1)}\right)
\end{array}\right.
$$

with $\mathbf{y}$ of the form:

$$
\mathbf{y}=\mathbf{h}\left(\mathbf{x}, \mathbf{u}, \dot{\mathbf{u}}, \ddot{\mathbf{u}}, \cdots, \mathbf{u}^{(\beta)}\right)
$$


The y output is called a flat output of the system (27), being necessary to have the same number of outputs than inputs (that is $y \in \mathbb{R}^{m}$ ).

It is easily seen that having found a flat output, it is possible to inverse the whole system. Path planning and trajectory tracking can be implemented, since the desired input $\mathbf{u}_{\mathrm{d}}(\mathbf{t})$ can be computed without any integretions according to the desired output $\mathbf{y}_{\mathbf{d}}(\mathbf{t})$ :

$$
\mathbf{u}_{\mathbf{d}}(\mathbf{t})=\mathbf{B}\left(\mathbf{y}_{\mathbf{d}}, \dot{\mathbf{y}}_{\mathbf{d}}, \ddot{\mathbf{y}}_{\mathbf{d}}, \cdots, \mathbf{y}_{\mathbf{d}}^{(\alpha+1)}\right)
$$

One of the great advantage of the method is that,from an initial desired trajectory, it is possible to calculate a corresponding control which take into account the internal dynamic of the system.

\section{B. Flatness of the system}

In order to find a flat output of model (26), a variable change, $\mathbf{x}=\boldsymbol{\Phi}(\mathbf{s})$, is performed as:

$$
\Phi \equiv\left\{\begin{array}{l}
\mathbf{x}_{1}=\mathbf{r} \\
\mathbf{x}_{2}=\theta \\
\mathbf{x}_{3}=\mathbf{v} \\
\mathbf{x}_{4}=\mathcal{T}(\theta) \cdot \omega
\end{array}\right.
$$

with $\mathcal{T}(\theta)$ defined in (9). We have also:

$$
\Phi^{-1} \equiv\left\{\begin{array}{l}
\mathbf{r}=\mathbf{x}_{1} \\
\theta=\mathbf{x}_{2} \\
\mathbf{v}=\mathbf{x}_{3} \\
\omega=\mathcal{T}\left(\mathbf{x}_{2}\right)^{-1} \cdot \mathbf{x}_{4}
\end{array}\right.
$$

The non singularity of $\mathcal{T}$ is always assured:

$$
\mathcal{T}^{-1}=\frac{1}{1+\delta^{2}}\left[\begin{array}{ccc}
1 & -\delta & 0 \\
\delta & 1 & 0 \\
-\beta & \beta \delta & 1+\delta^{2}
\end{array}\right]
$$

and its first time derivative is given by:

$$
\begin{aligned}
\dot{\mathcal{T}}(\theta) & =\left[\begin{array}{ccc}
0 & \dot{\delta} & 0 \\
-\dot{\delta} & 0 & 0 \\
\dot{\beta} & 0 & 0
\end{array}\right]=\left[\begin{array}{ccc}
0 & r+\beta p & 0 \\
-r-\beta p & 0 & 0 \\
q-\delta p & 0 & 0
\end{array}\right] \triangleq \\
& \triangleq \mathcal{P}(\theta, \omega)
\end{aligned}
$$

so:

$$
\dot{T}\left(\mathbf{x}_{2}\right)=\mathcal{P}\left(\mathbf{x}_{2}, \mathcal{T}\left(\mathbf{x}_{2}\right)^{-1} \cdot \mathbf{x}_{4}\right)
$$

Therefore, new model is given by:

$$
\left\{\begin{aligned}
\dot{\mathbf{x}}_{1}= & \mathbf{x}_{3} \\
\dot{\mathbf{x}}_{2}= & \mathbf{x}_{4} \\
\dot{\mathbf{x}}_{3}= & \frac{1}{m} \mathrm{~V}_{1}\left(\mathbf{x}_{1}, \mathbf{x}_{2}\right)+\frac{1}{\mathrm{~m}} \mathcal{K}_{\mathbf{f}}^{\mathbf{C}} \cdot \mathbf{u} \\
\dot{\mathbf{x}}_{4}= & \mathcal{P}\left(\mathbf{x}_{2}, \mathcal{T}\left(\mathbf{x}_{2}\right)^{-1} \cdot \mathbf{x}_{4}\right) \cdot \mathcal{T}^{-1}\left(\mathbf{x}_{2}\right) \cdot \mathbf{x}_{4}+ \\
& +\mathcal{T}\left(\mathbf{x}_{2}\right) \cdot \mathcal{M}\left(\mathbf{x}_{2}\right) \cdot \mathbf{V}_{2}\left(\mathbf{x}_{1}, \mathbf{x}_{2}\right)- \\
& -\mathcal{T}\left(\mathbf{x}_{2}\right) \cdot \mathcal{M}\left(\mathbf{x}_{2}\right) \cdot \mathcal{N}\left(\mathbf{x}_{2}, \mathcal{T}^{-1}\left(\mathbf{x}_{2}\right) \cdot \mathbf{x}_{4}\right) \\
& \mathcal{T}^{-1}\left(\mathbf{x}_{2}\right) \cdot \mathbf{x}_{4}+\mathcal{T}\left(\mathbf{x}_{2}\right) \cdot \mathcal{S}\left(\mathbf{x}_{2}\right) \cdot \mathbf{u}
\end{aligned}\right.
$$

State vector is now grouped as follows:

$$
\xi_{1} \triangleq\left[\begin{array}{l}
\mathbf{x}_{1} \\
\mathbf{x}_{2}
\end{array}\right] \quad \xi_{2} \triangleq\left[\begin{array}{l}
\mathbf{x}_{3} \\
\mathbf{x}_{4}
\end{array}\right]
$$

then model is written as:

$$
\left\{\begin{array}{l}
\dot{\xi_{1}}=\xi_{2} \\
\dot{\xi_{2}}=\mathcal{F}\left(\xi_{1}, \xi_{2}\right)+\mathcal{G}\left(\xi_{1}\right) \cdot \mathbf{u}
\end{array}\right.
$$

with

$$
\begin{aligned}
& \mathcal{F}\left(\xi_{1}, \xi_{2}\right) \triangleq\left[\begin{array}{c}
\frac{1}{m} \mathbf{V}_{1}\left(\xi_{1}\right) \\
\mathcal{T}\left(\xi_{1}\right) \cdot \mathcal{M}\left(\xi_{1}\right) \cdot \mathbf{V}_{2}\left(\xi_{1}\right)
\end{array}\right]+ \\
& +\left[\begin{array}{c}
\mathbf{0}_{3} \\
\dot{\mathcal{P}}\left(\xi_{1}, \xi_{2}\right) \cdot \mathcal{T}^{-1}\left(\xi_{1}\right)-\mathcal{T}\left(\xi_{1}\right) \cdot \mathcal{M}\left(\xi_{1}\right) \cdot \mathcal{N}\left(\xi_{1}, \xi_{2}\right) \cdot \mathcal{T}^{-1}\left(\xi_{1}\right)
\end{array}\right] \cdot \xi_{2}
\end{aligned}
$$

and

$$
\mathcal{G}\left(\xi_{1}\right) \triangleq\left[\begin{array}{c}
\frac{1}{m} \mathcal{K}_{\mathbf{f}}^{\mathbf{C}} \\
\mathcal{T}\left(\xi_{\mathbf{1}}\right) \cdot \mathcal{S}\left(\xi_{\mathbf{1}}\right)
\end{array}\right]
$$

$\mathrm{V}_{\mathbf{I}}$ and $\mathrm{V}_{\mathbf{2}}$ vectors, $\mathcal{M}, \mathcal{N}, \mathcal{K}_{\mathrm{f}}^{\mathbf{C}}$ and $\mathcal{K}_{\mathrm{m}}^{\mathrm{C}}$ matrices being defined in section II-D and $\mathbf{0}_{\mathbf{3}}$ a null dimension 3 column vector

The vector $\xi_{1}$ represents the "position" of the shaft, $\xi_{1}=$ $\left[\begin{array}{llllll}x & y & z & \alpha & \beta & \delta\end{array}\right]^{\mathbf{T}}$ while the vector $\xi_{2}$ represents its "velocity" since it contains the Euler angles derivatives, $\xi_{2}=\left[\begin{array}{llllll}v_{x} & v_{y} & v_{z} & \dot{\alpha} & \dot{\beta} & \dot{\delta}\end{array}\right]^{\mathbf{T}}$.

\section{B.1 Flat output}

A possible choice of the flat output is $\mathrm{z}=\xi_{1}$. Since we can express the states and the controls of the system in terms of $(\mathbf{z}, \dot{\mathbf{z}}, \ddot{\mathbf{z}})$, the flatness property is true for $\mathbf{z}$. Precisely:

$$
\xi_{1}=\mathbf{z} \quad, \quad \xi_{2}=\dot{\mathbf{z}}
$$

and

$$
\mathbf{u}=\mathcal{G}^{-1}(\mathbf{z}) \cdot(\ddot{\mathbf{z}}-\mathcal{F}(\mathbf{z}, \dot{\mathbf{z}}))
$$

Due to the whole system complexity, only numerical and experimental tests have been performed in order to proof the non singularity of $\mathcal{G}$ (the symbolic computational toolbox Mapple is not able to compute this problem). Then, desired control path can be computed in function to a desired path output:

$$
\mathbf{u}_{\mathbf{d}}=\mathcal{G}^{-1}\left(\mathbf{z}_{\mathbf{d}}\right) \cdot\left(\ddot{\mathbf{z}}_{\mathbf{d}}-\mathcal{F}\left(\mathbf{z}_{\mathrm{d}}, \dot{\mathbf{z}}_{\mathbf{d}}\right)\right) \triangleq \Psi(\mathbf{z}, \dot{\mathbf{z}}, \ddot{\mathbf{z}})
$$

\section{Path planning}

Polynomial trajectories for output $\mathbf{z}$ are chosen for simulations in order to implement continuous operational trajectories as specified by the final user. Further studies should consider different trajectories generation in order to improve current performances or avoiding their saturations. 
A polynomial trajectory $t \rightarrow z_{j}(t)$ for all $j=1, \ldots, m$, which assures continuity of output first and second derivatives, is such that [2]:

$$
z(t)=a_{0}+\sum_{k=1}^{5} a_{k}\left(\frac{t-t_{i}}{t_{f}-t_{i}}\right)^{k}
$$

Where $a_{k}$ parameters are computed with initial and final conditions in $\mathbf{z}$ :

$$
\begin{array}{ll}
\mathbf{z}\left(\mathbf{t}_{\mathbf{i}}\right)=\mathbf{z}_{\mathbf{i}}=\mathbf{a}_{\mathbf{0}} & \mathbf{z}\left(\mathbf{t}_{\mathbf{f}}\right)=\mathbf{z}_{\mathbf{f}} \\
\dot{\mathbf{z}}\left(t_{i}\right)=\mathbf{0} & \dot{\mathbf{z}}\left(t_{f}\right)=0 \\
\ddot{\mathbf{z}}\left(t_{i}\right)=\mathbf{0} & \ddot{\mathbf{z}}\left(t_{f}\right)=0
\end{array}
$$

In the presented study, the desired trajectories are always known before to start. That implies that an off-line calculation of the polynomials can be made.

\section{Linear feedback}

One of main disadvantages of system inversion using flat control is that model dynamics and model parameters must be known with a good level of precision. In order to face parameters uncertainties or even measurement noise, a linear feedback is implemented to compute corrections needed in control variables in function to the difference between actual output and desired output (error signal). Figure 2 shows the final control law scheme.

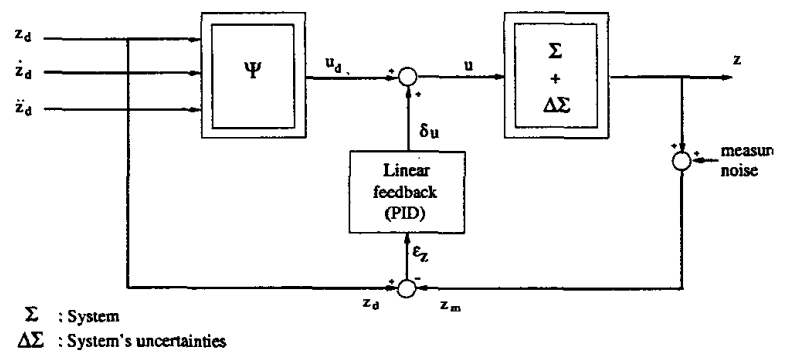

Fig. 2. System control loop

\section{Simulations}

The system (26) and the designed control law of figure 2 have been implemented for simulation in Matlab-Simulink environment. Linear feedback is formed by 6 independent PID controllers experimentally adjusted. Random parametric variations are applied at inertia matrix elements $\left(\mathcal{I}_{\mathrm{b}}\right)$ and at negative stiffness force constants $\left(k_{i}^{S}\right)$.

Top figure $3 \mathrm{a}$ ) shows desired 5 degrees polynomial scan angle waveform choose to implement a continuous function for a step response from an initial position at $-\frac{\pi}{2}$ to a final postion at 0 . The figure 3.b is the difference between this desired angle and output angle, thus error output. The figures 3.c 3.d and contain parasite angles evolution being always of $10^{-7} \mathrm{rad}$ order for $\beta$ and of $10^{-7} \mathrm{rad}$ order for $\delta$.

Figure 4 contains control currents time evolution.

Same kind of simulations are repeated adding random external perturbation forces acting on the rotor shaft. Figure 5 a) illustrates desired scan angle and output scan angle
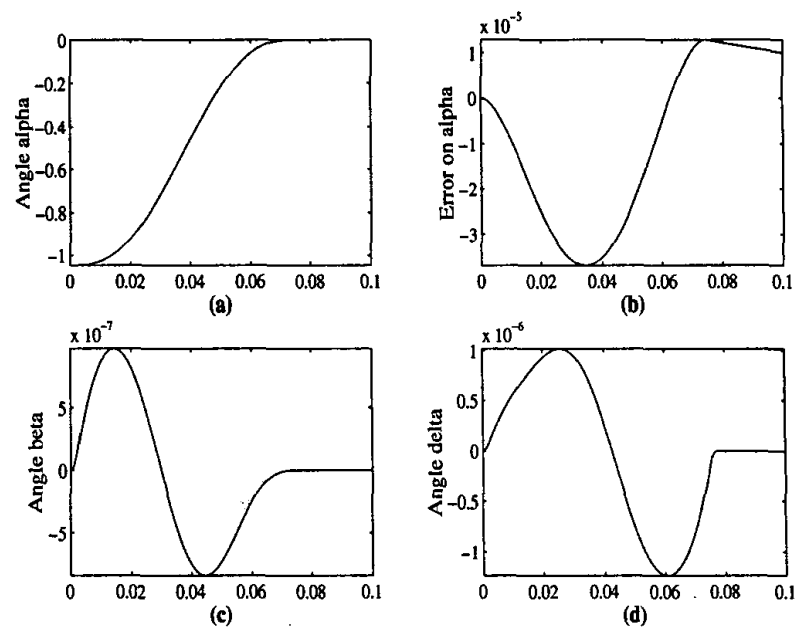

Fig. 3. Simulation in nominal condition
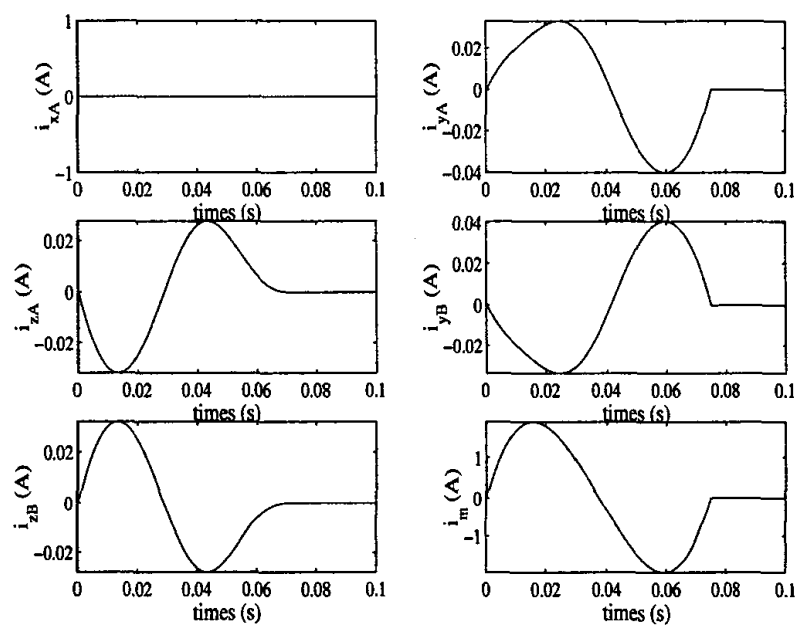

Fig. 4. Control currents

error, and figure $5 \mathrm{~b}$ ) contains parasite angle time evolution.

In both simulations desired angle error and parasite angles are always below $10^{-5} \mathrm{rad}$, which satisfies system precision performances.

\section{CONClusions AND PERSPECTIVES}

In this paper is shown a mechanical modeling for a semi active magnetic bearing based system. It is also shown how non linearities of the model are being taken into account for trajectory tracking using flat control and how parametric uncertainties and noise are considered as well using a linear feedback. The non linear system and its associated control law are simulated in a Matlab-Simulink environment proving that the proposed control law reaches system specifications. Further research should study more precisely the 

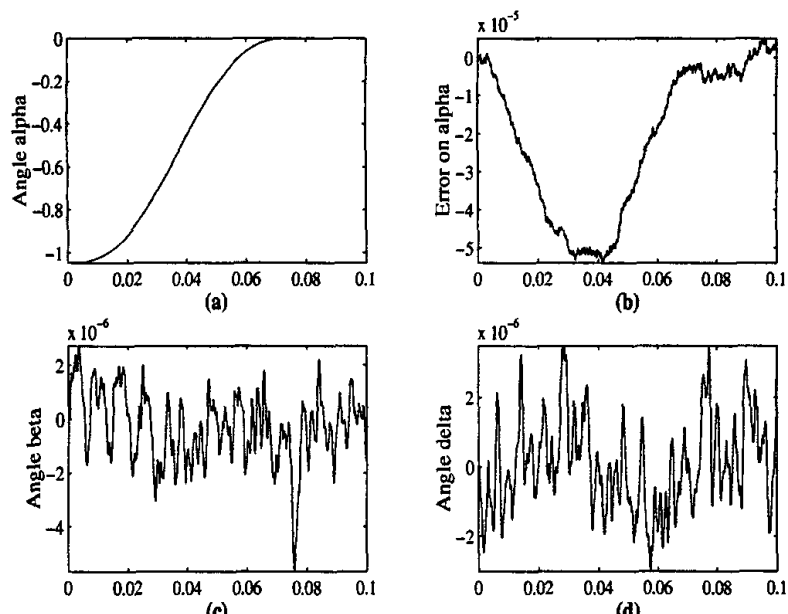

Fig. 5. Simulation with force perturbations applied

linear feedback (6 PID in parallel experimentally adjusted are used in this paper) as well as the trajectories generation (polynomial trajectories used) in order to improve system performances. In a practical approach, quantification noise effects and measurement noise should be taken into account too.

\section{REFERENCES}

[1] M. Fliess, J. Levine, P. Martin, and P. Rouchon. Flatness and defect of non linear systems. introductory theory and examples. Int. Journal of Control, 61:1327-1361, 1995.

[2] M. Fliess, J. Levine, P. Martin, and P. Rouchon. Système différentiellement plats : intérêts et exemples. In Ecole d'été d'Automatique : Flat systems, theory and practice, Grenoble, 1996.

[3] A. Isidori. Non linear control systems. Springer, New York, 1995.

4] J. Levine, J. Lottin, and J.C. Ponsart. Non linear approach to the control of magnetic bearings. IEEE Trans on Control Systems Technology, 5:524-544, 1996.

[5] H. Nijmeijer and A. J. Van der Schaft. Dynamical control systems. Springer, New York, 1995.

[6] M. Sidif. Spacecraft Dynamics \& control. A practical enginnering approach. Cambridge Aerospace series 7, London, 1997. 